The $8^{\text {th }}$ International Conference of the Slovenian Society for Non-Destructive Testing

"Application of Contemporary Non-Destructive Testing in Engineering"

September 1-3, 2005, Portorož, Slovenia, pp. 391-399

\title{
EXPERIMENTAL APPROACHES TO CREEP-INDUCED DAMAGING EFFECTS UNDER NORMAL AMBIENT AND TRANSIENT MOISTURE CONDITIONS IN A GFR POLYMER COMPOSITE
}

\author{
V.N. Kytopoulos, I.N. Prassianakis, E. Sideridis \\ National Technical University of Athens, Faculty of Applied Mathematics and Physics Sciences, Department of \\ Mechanics, Laboratory of Strength and Materials, Iroon of Polytechniou Avenue 5, GR-157 73, Zografou, \\ Athens, Greece, E-mail: prasian@central.ntua.gr
}

\begin{abstract}
In this study some Damaging Effects induced by creep loading under normal ambient as well as transient moisture conditions i.e. by the so-called mechano-sorptive creep in a certain glass fibre-reinforced (GFR) polyester matrix composite are investigated by means of two basic types of experimental approaching techniques. The first type of these techniques was based on the influence of the creep-induced damaging effect on some relevant mechanical parameters such as elastic modulus and fracture stress, whereas the second type on the influence on a relevant physico-chemical parameter such as moisture diffusivity out of the material. By the above approaches it was shown that despite the non-neglecting scatter in the experimental data certain irresistible general trends might be assessed. In this context it was found that the damage "response" is markedly sensitive to the respective applied "detection" technique and therefore the damage evolution data obtained by these different techniques are not comparable.

By comparison between the applied techniques it seems reasonable to assume that the "diffusional" technique due to its inherent microscopic mass transport processes in form of $\mathrm{H}_{2} \mathrm{O}$ molecules diffusion and therefore its ability to "detect" microstructural detects on a much lower scale can give a better approach to the damage accumulation phenomena.
\end{abstract}

Keywords: Creep, Diffusivity, Moisture, Damage, NDT, Microcraking, Mechanical parameters

\section{Introduction}

It is well known that materials subjected to stresses well below that required for macroscopic failure undergo deterioration of their structure. The deterioration of the material structure is caused by nucleation and growing-size accumulation of internal defects or discontinuities such as microcracks and voids/cavities fact, which implies related "internal" decrease in load carrying area. In this sense material damage is defined as the property whereby the material strength diminishes before failure occurs. In recent years there has been considerable effort to study the types of the so-called "material damage" and its evolution and to correlate it with adequate mechanical as well as physical properties of the material. 
In this sense the first basic problem is how to define and adopt the most adequate damage variables; i.e. what kind of real physical nature these variables should have in order to represent such damage state property. The second basic complex problem, on the other hand is how to quantity the magnitude of these variables [1].

In this direction certain physical techniques such as sonic inspection have a very long tradition in engineering practice. The presence of macroscopic cracks influence the resonance frequencies and damping quite strongly and human ear may often be used to detect such major cracks. In contrast, micro-cracks, voids and pores, which constitute material damage may not be detected or measured by audible sonic (acoustic emission) methods. Ultrasonic techniques may be, however, be used for this scope in various ways with varying accuracy. One particular experimental ultrasonic approach is for example that proposed recently [2], where the material damage can be evaluated quickly and with higher accuracy than other ultrasonic techniques by measuring only changes of signal attenuation. In this context it should be also mentioned the paper [3] where by a similar ultrasonic technique the moisture-induced internal damage is evaluated in a good manner.

Recently, a novel non-destructive approaching technique has been proposed [4-6] for the damage evaluation in certain polymer composites. This technique is based on the net effects of the internal structural changes, on the moisture diffusivity of the material. It has been shown that this thermophysical approaching technique can be used in a complementary way to study the damage and can provide a powerful tool to gain a deeper insight into the general phenomenon of damage evolution. In this sense it would be valuable to try to compare the above mentioned thermophysical (diffusional) technique with a non-physical i.e. a quasi non-destructive mechanical one such as stiffness reduction and a destructive one such as fracture stress reduction.

The purpose of this paper at first, to investigate by means of these three techniques the evaluation of creep-induced damage, under normal ambient and transient moisture conditions in a certain polymer composite, and the arising coupling effects between creep damage and the moisture-induce damage. Then, to compare and to discuss the response of these techniques on the material damage.

\section{Damage Definition}

Damage can be defined as the effective surface density of micro-cracks and cavities in any plane of a representative volume element. There are a number of different methods of measuring the damage. The definition of damage in the continuum mechanics context [1] is given as follows: If $n$ is the normal which defines a surface of intersection $d S$ of a volume element $\delta \mathrm{V}$ and if $\delta S_{0}$ is the effective surface of intersection of micro-cracks and micro-cavities with the plane of $d S$, the damage is defined by the expression:

$$
D_{(n)}=\frac{\delta S_{0}}{\delta S}
$$

thus when $D_{(n)}=0$ there is no damage in the direction $n$, while when $D_{(n)} \rightarrow 1$ the element breaks into two parts along the plane $n$.

For a bar subjected to a tensile load (when damage micro-cavities are created within the specimen as voids, micro-cracks etc), let its apparent area of cross-section be $A_{\alpha}$, the area occupied by micro-cavities be $A_{d}$ and the effective cross-sectional area be $A_{\varepsilon}$. Then, the continuous damage parameter $D$ may be defined as: 


$$
D_{k}=1-\frac{A_{\varepsilon}}{A_{\alpha}}
$$

where, when $A_{\alpha}=A_{\varepsilon}, A_{d}=0, D_{k}=0$ there is no-damage, while when $A_{\alpha}=A_{d}, A_{\varepsilon}=0$, $D_{k}=1$ there is damage. Based on the definitions of damage, expressed by the formulae (1) and (2) and according to the adopted experimental approach several measuring formulae for the damage parameter $D$ can be derived.

\section{Damage measures and related techniques}

\subsection{Elastic Modulus}

Considering several most common techniques to evaluate damage (ultrasonic waves, cyclic softening and damping, electrical resistance, elastic modulus i.e. stiffness reduction) the most appropriate one for damage evaluation is the measurement of the tensile elastic modulus. This technique is derived directly from the coupling between elasticity and damage and is deduced from the principle of strain equivalence [1]:

$$
\tilde{\sigma}=\frac{\sigma}{1-D}=E \cdot \varepsilon_{e}
$$

or

$$
\sigma=E(1-D) \cdot \varepsilon_{e}
$$

where $E \times(1-D)=\tilde{E}$ may be interpreted as the elastic modulus of the damaged material. If the $E_{0}$, the Young's modulus of the virgin, undamaged, material is known, and $\tilde{E}_{i}$ can be measured, the damage is defined as:

$$
D_{i}=1-\frac{\widetilde{E}_{i}}{E_{0}}
$$

where " $i$ " denotes the number (steps) of damaging loads. It must be mentioned that the measurement of $\tilde{E}$ is often difficult due to: first the localization of damage which requires the use of very small strain gauges, second the small non-linearities which always exist in the elastic range even during unloading and third micro-plasticity phenomena which, usually neglected, must be considered when damage occurs in the elastic modulus range, even if macroscopic plastic strain does not exist. Because of such elastic-induced microplastic structural changes the stiffness (elastic modulus) degradation measurement may represent in fact a quasi nondestructive technique.

\subsection{Fracture stress}

Bearing in mind the foregoing and those mentioned concerning eq. (2) one can define a (destructive) Damage measure as follows:

$$
D_{i}=1-\frac{\sigma_{i}}{\sigma_{0}}
$$

where $\sigma_{i}$ and $\sigma_{0}$ are the (real) fracture stress for damaged and for undamaged (virgin) material respectively. 


\subsection{Moisture Absorption/Desorption}

General Aspects. Although a large number of papers concerning the thermomechanical degradation due to the moisture absorption/desorption can be found, little work has been done up to now, concerning the combined material characterization in terms of microstructural changes or damage degree induced by mechanical loading as well as by moisture absorption. In reality, many composite structures experience a changing moisture environment (outdoor structures) and therefore the mechanical response of hydrophilic materials concurrently undergoing moisture sorption/desorption i.e. the so-called mechano-sorptive research may be of great interest. Despite the relative extended mechano-sorptive research on wood, and, to a less extent on polymeric materials very little reference has been found on composite materials $[7,8,10]$.

Moisture Diffusivity. The Diffusion phenomenon, in general, represents certain thermodynamicpassive mass/molecular transport processes in the materials. This microscopic behaviour of the material is macroscopically determinate by its (mass). Diffusivity, “ $\widetilde{D}$ ” which is a measure of the speed by which the moisture concentration changes inside the material. It was found that many transport phenomena can be described by means of the first (linear) and second (nonlinear) law of the Fickian diffusion mechanisms from which the corresponding mass Diffusivity, $\tilde{D}$, can be calculated [9]. Moreover, the linear Fickian law plays a very import role because of its relative simplicity in the calculation of the formulae of diffusivity $\widetilde{D}$. This, in turn, can be measured by the standard experimental procedure given elsewhere $[9,10]$ and concerning relative simple moisture absorption/desorption test. This is a usual test for material characterization, which can be made in terms of thermomechanical as well as viscoelastic behaviour [11]. We can define a diffusional damage measure in a similar way to eqs (4) and (5) which is given as:

$$
D_{i}=1-\left(\frac{\tilde{D}_{i}}{\tilde{D}_{0}}\right)^{n}
$$

with $n=1$ if $\tilde{D}_{i} \leq \widetilde{D}_{0}$ and $n=-1$ if $\tilde{D}_{i} \geq \widetilde{D}_{0}$, where $\tilde{D}_{0}$ and $\tilde{D}_{i}$ are the corresponding mass diffusivity of the virgin and mechanically damaged material respectively.

\section{Experimental procedure}

\subsection{Mechanical procedure}

The stiffness reduction testing procedure, to obtain the elastic modulus damage measure, was performed on an Instron-type loading machine by applying a quasistatic strain rate of about $10^{-4} / \mathrm{s}$. The elastic modulus was measured by means of adequate electrical strain gauges. The creep loadings were conducted at a constant nominal stress level of about $\sigma_{f} / 2 \cong 4,5 \mathrm{kp} / \mathrm{mm}^{2}$, which from preliminary estimations was high enough to have satisfactory damage accumulation but low enough to break the material within the desired experimental time range. All creep loading experiments were carried out at ambient temperature of about $\approx 25^{\circ} \mathrm{C}$. From preliminary experiments it was found that the moisture content of the material during the time of creep loading has changed up to $50 \%$ from its initial state of saturation. In this aspect one should assume transient moisture condition of creep-induced damage and try to explain the phenomena by means of the so-called mechano-sorptive damage effects. The tensile loading experiment up to fracture was performed at a constant strain rate $\approx 10^{-4} / \mathrm{s}$. The material used was a random fibre reinforced polyester matrix composite with a volume fraction of fibres of about $15 \%$. 


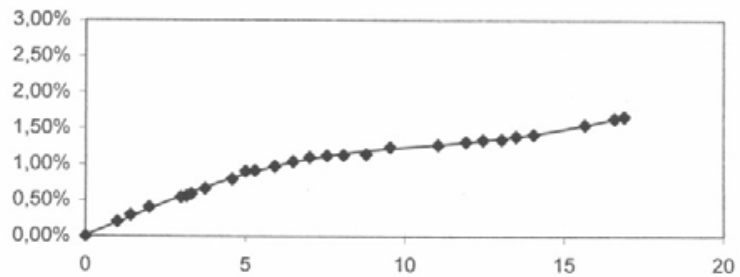

Fig. 1: Percentage moisture absorption by mass versus time, $t^{1 / 2}$ obtained at $20^{\circ} \mathrm{C}$.

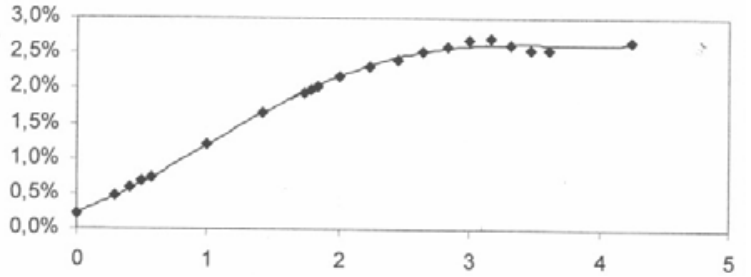

Fig. 2: Percentage moisture absorption by mass vs. time, $t^{1 / 2}$, obtained at $80^{\circ} \mathrm{C}$.

\subsection{Moisture Absorption procedures}

As mentioned previously the saturating absorption experiments were performed following the test procedures given elsewhere [9,10]. Especially in this paper we have try to shorten the testing time by using "high" water temperature of about $80^{\circ} \mathrm{C}$, which was significantly lower than the glass transition temperature $T_{g} \cong 115^{\circ}$ of the composite material. In fact, Figs. (1) and (2) show the relative great difference between the moisture absorption behaviour at low temperature of $\approx 20^{\circ} \mathrm{C}$ and "high" temperature of $\approx 80^{\circ} \mathrm{C}$ respectively. In this way the saturation time (in days) required for each tested specimen was shortened by about 10 times. Moreover, by means of preliminary experiments it was shown that possible high temperature hygrothermal damaging effects such as osmotic degradations, surface blistering, surface peeling, resin leaching and dissolution (mass loss effects), surface microcracking and other intrinsic physical damage may be minimized but not totally avoided.

\section{Results and Discussion}

The Figs. (3) and (4) show the curves of the damage evolution with time under normal ambient and transient moisture conditions respectively evaluated by means of the elastic modulus (stiffness) reduction technique whereas Figs. (5) and (6) show the damage evolution with time under the same conditions mentioned above but evaluated by the fracture stress reduction technique.

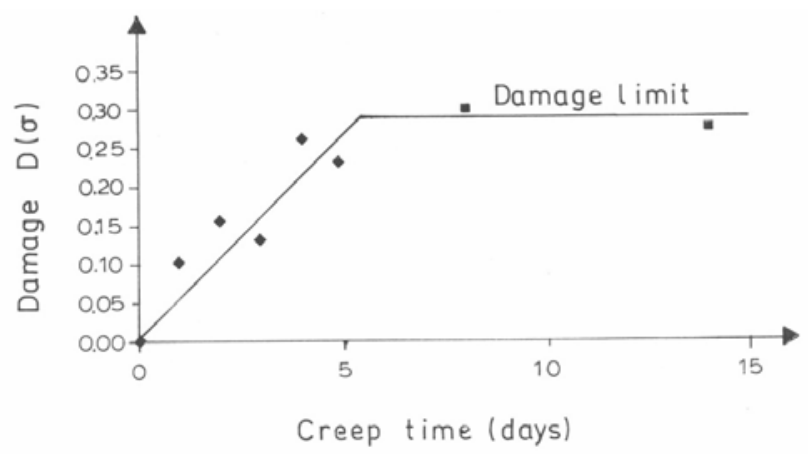

Fig. 3: Damage evolution with creep time for normal ambient conditions obtained by stiffness reduction test.

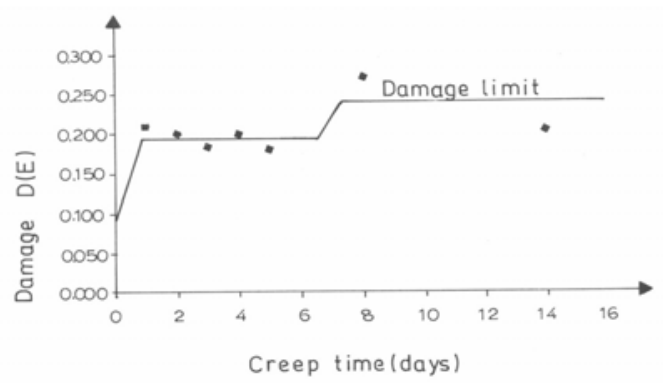

Fig. 4: Damage evolution with creep time for transient moisture conditions obtained by stiffness reduction tests.

Despite the non-negligible data scatter by the comparison of these figures to each other, one can distinct certain common characteristic trends. First, the higher initial damage value for transient conditions and second the tendency for attaining asymptotically a damage "saturation" level (limit) after a certain time of creep loading. Furthermore, one can distinct the differences 
between the damage limit values for the two above techniques. The value of this limit is greater in the case of fracture stress measure compared to that obtained by the elastic modulus measure technique. Also, one can distinct the different initial damage value of these techniques: it is higher in the case of fracture stress measure.

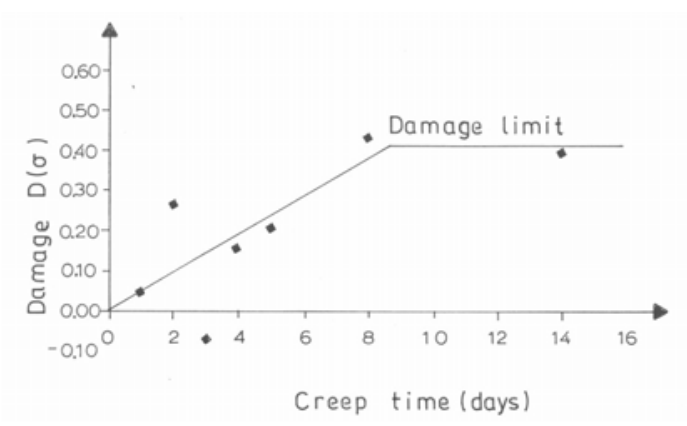

Fig. 5: Damage evolution with creep time for normal ambient conditions obtained by fracture stress reduction test.

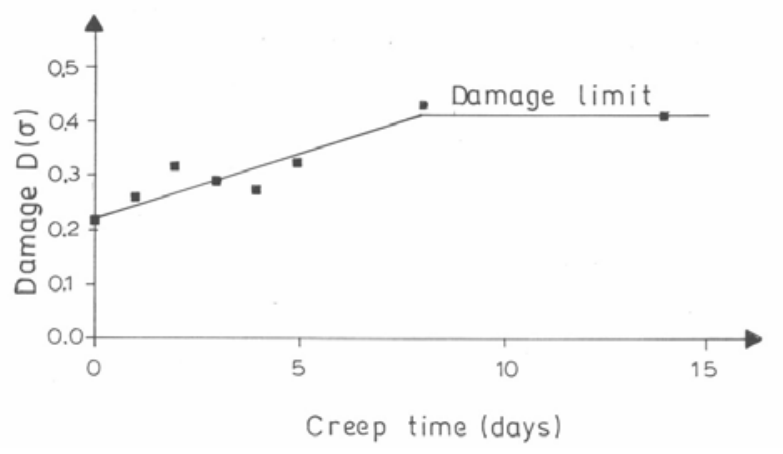

Fig. 6: Damage evolution with creep time for transient moisture obtained by fracture stress reduction measure.

The above mentioned common trend of creation of a limiting damage could be attributed to the fact that the evolution of internal state of the creep loaded material appears mainly by two classes of microstructural change: one is strain hardening related to viscoelastic molecular chain rearrangements and the other is internal damage characterized by formation of distributed microcavities or cracklike cavities and microcracks. In other words the creep damage involves not only the nucleation and growth of (micro) cavities and (sub) microcracks, but also their coupling with the strain hardening of the matrix material or with strain localization due to relative large deformations. The latter may cause a macromolecular orientation-induced strain hardening in the immediate vicinity of the raisers, which leads to failure blunting effects and in this way to a net damage stabilization.

The initial (non-zero) damage observed after creep under transient moisture conditions is clearly related to the corresponding initial material degradation due to the saturating moisture absorption i.e. to constant moisture-induced mechanical degradation.

The higher value of the damage limit measured by fracture stress testing compared to those measured by stiffness reduction testing is due to the different evolution phases of the damage accumulation during the finite testing time. This means that the material tested to fracture has a different stress (strain) history compared to the elastic tested material in the sense that in the first case an additional (new) elastic-plastic microcracking may be induced and at the same time the primary (“old”) cracklike cavities may coalesce in reducing the net load carrying area.

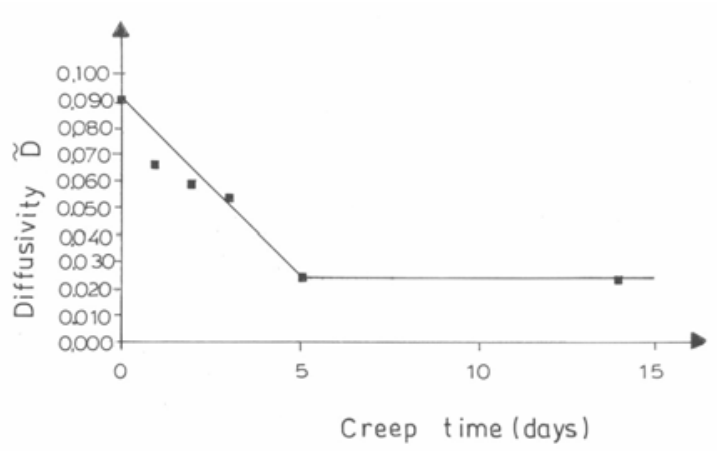

Fig. 7: Diffusivity change with creep time for normal ambient conditions.

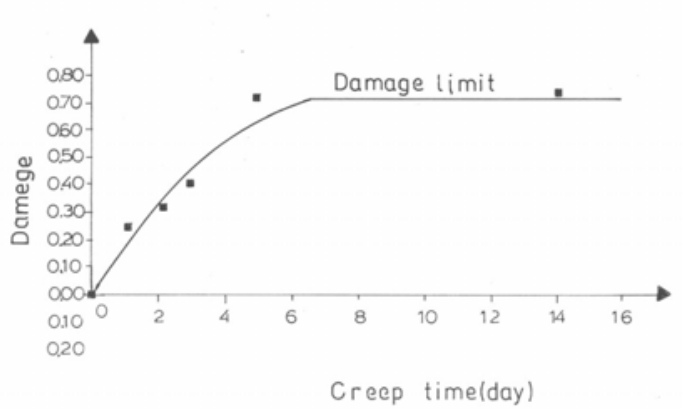

Fig. 8: Damage evaluation with creep time for normal ambient conditions obtained by diffusional measure. 


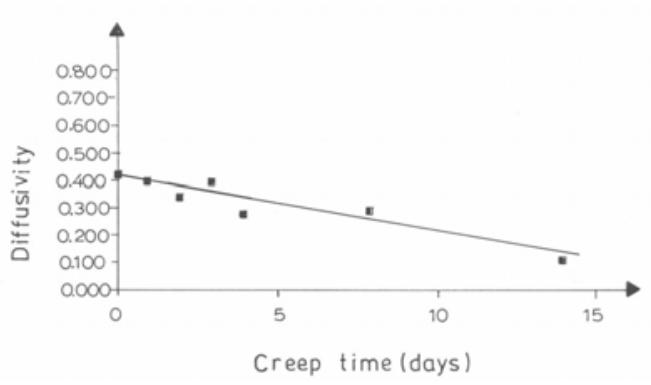

Fig. 9: Diffusivity change with creep time for transient moisture conditions.

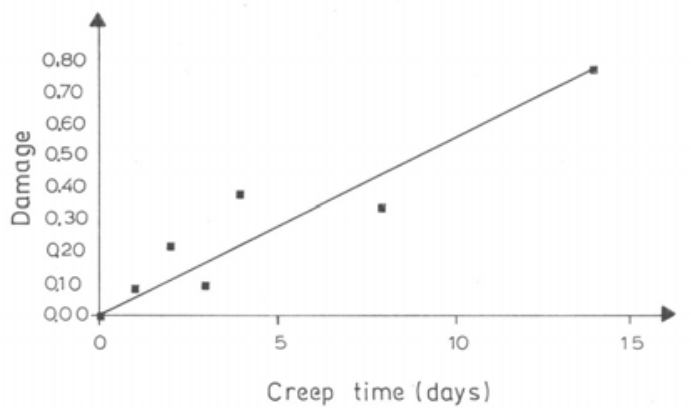

Fig. 10: Damage evolution with creep time for transient moisture conditions obtained by diffusional measure..

The data shown in the series of Figs. (7-10) are related to the damage evaluation and characterization by using the moisture absorption/desorption test procedure. One can observe the decrease of the diffusivity with time of creep for normal as well as transient conditions (Figs. 7,9). In order to be conform with the positive value of diffusional damage given by eq. (8) this unexpected behaviour forced us to take $n=+1$ in this equation and to construct in this way the graphs of damage evolution shown in Fig. (8) and (10). At this stage it must be pointed out that the moisture absorption behaviour of the materials has not obeyed an ideal linear Fickian diffusion law. In order to calculate the Diffusivity by means of this law one has to approximate the real (non-Fickian) behaviour by a ideal linear Fickian. This was done by means of the statements given in [11], where according to them any (not too strong) non-Fickian (anomalous) moisture absorption can be roughly approximated or simulated by a lower and/or upper linear Fickian limit as shown in Fig. (11).

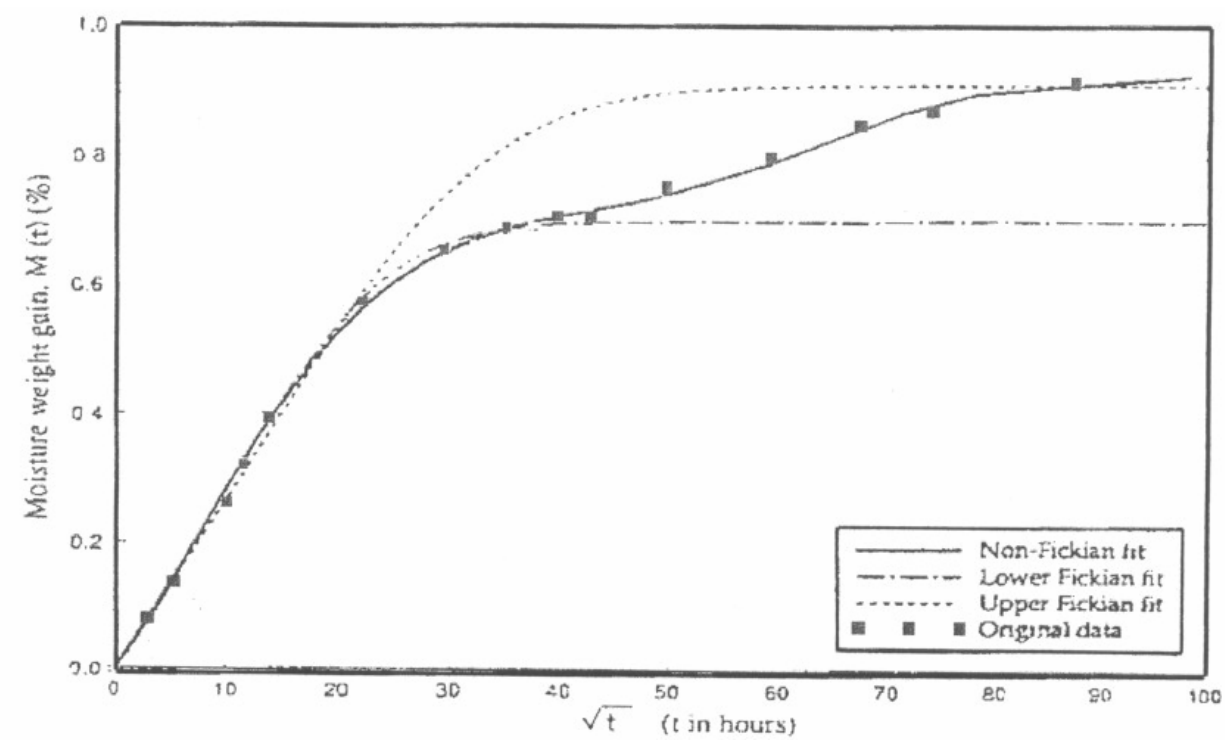

Fig. 11: Interpolating Approximation of the non-Fickian absorption behaviour [11].

Because of the easier experimental determination of the upper saturation level we have chosen the related upper Fickian limit approximation by applying the simplest Fickian formula for the Diffusivity calculation as given:

$$
D=\left(\frac{\pi}{16 h^{2}}\right) \cdot\left(\frac{F}{m_{\infty}}\right)^{2}
$$


where $m_{\infty}$ is the equilibrium, upper saturation level for a particular environment and $F$ the initial slope of a plot of moisture $m(t)$ as a function of time $t^{1 / 2}$ and $h$ the specimen thickness. It is well established that, in general, the Diffusivity should increase with damage because of the increased number of diffusion pathways created by the damage mechanisms [12,13]. As mentioned above this is in contrast with our experimental findings which means that damage should have a "negative" effect on the moisture diffusion in the material. This could be tentatively explained by assuming the existence of "active" damage, which acts as "paths" of molecular transport as well as "passive" damage, which acts as "barriers" to molecular transport. This in turn means that "active" damage may be represented by "open" microcracks whereas the "passive" damage by "closed" microcracks. Now, during creep loading the damage created can be of elastic/unelastic and/or plastic/viscous type. Upon unloading (creep termination) the termination damage state is "freezed in" where the first type of damage is being "closed" whereas the second kind remains "open". Depending on the material ductility (low order high) the "content" of the two type of damage can vary, fact which means that for our elastic-brittle material (ductility $\approx 2-3 \%$ ) the "closed" type damage is dominating. During "closing" the microcracks/voids may entrap $\mathrm{H}_{2} \mathrm{O}$ molecules by acting as water traps after termination of mechano-sorptive test.

Now, during the high temperature $\left(\approx 80^{\circ} C\right)$ moisture desorption test the water in the polymer matrix material is at a thermodynamic-molecular equilibrium of a liquid-vapor phase. The water diffusion through the undamaged matrix regions obeys to a linear Fickia law whereas the diffusion through the damage regions obeys mainly a non-Fickian law $[11,12,13]$. This means that any deviations of the moisture desorption curve from its Fickian shape should be attributed to damage formation in the material. In this context the non-Fickian deviations can be caused by two coupling effects: first the entrapped water phase cannot "escape" to easy (bound phase) and so its net Diffusivity out of the matrix is lowered; second the unbound phase existing in the free volume can with difficulty diffuse out of the matrix due to the "obstacles" in form of "twodimensional" discontinuities formed by the (elastically) "closed" microcracks (transport or diffusion barrier effect).

In contrast to the two foregoing "mechanical" techniques of damage measure, the diffusional technique presents a very different response to the damage. These differences in the response behaviour are expressed by a higher limit, Fig. (8) or ultimate damage, Fig. (10), and, in part, by no-tendency of limit damage formation, Fig. (10). It is clear that the diffusional technique, based on its inherent microphysical (molecular) transport phenomena, has a much higher damage response or detection sensitivity fact that explains the above first different behaviour. Mechanosorptive damaging effects are much stronger than those of ambient creep conditions fact, that by considering the higher detection sensitivity may explain the above second difference in the response behaviour.

\section{Conclusions}

The present investigation of creep-induced damaging effects under normal ambient and transient moisture conditions made by three different techniques has shown that the diffusional technique based on the moisture Diffusivity measurement should have a much higher damage detection sensitivity compared to the two other mechanical techniques based on the elastic modulus and fracture stress reduction measurements. It was also shown that in most of the applied techniques a tendency for the formation of a limiting damage level can be stated. The Damage values measured by moisture absorption were greater than the values obtained by the mechanical techniques. In general the two mechanical techniques give similar data compared to the diffusional where the damage evolution is different In this context "anomalous" damage effects on the moisture desorption profile such as the Diffusivity reduction with creep time have been 
tentatively explained by means of a proposed "transport barrier" mechanism. By means of the three applied measure techniques it was possible to show that the so-called mechano-sorptive damaging effects induced by creep under transient moisture conditions are much stronger than those induced by creep under normal ambient conditions. Taking into consideration the relative great differences in the physical basis between the mechanical and the thermophysical (diffusional) technique the obtained data can be seen to be in satisfactory agreement fact which means that the proposed non-destructive moisture absorption technique may be used as complementary one for such studies.

\section{References}

[1] Lemaitre J.: Formulation and identification of damage kinetic constitutive equations, in: Continuum Damage Mechanics, Theorie and Applications (Krajcinovic D., Lemaitre J. eds.), Springer Verlag Wien-New York, 1987, 39-87.

[2] Prassianakis, N.N.: An experimental approach to damage evaluation using ultrasounds, European Journal of NDT, Vol. 3, No. 3, 1994, 93-96.

[3] Lee B.L., Yang T.W.: Moisture Effects on Isobutylene-Isoprene Copolymer-Based Compsite Barrier. I: Moisture diffusion and detection, Polymer Engineering and Science, Vol. 36, No. 9, 1996, 1217-1231.

[4] Kytopoulos V.N., Papadopoulos G. About moisture absorption effects in certain epoxy composite systems, Proceedings of the International Symposium of Multisaling in Mechanics, Greece, 2002, 228-238.

[5] Prassianakis I.N., Sideridis E., Kytopoulos V.N., Prassianakis N.I., Papapetrou V.: Destructive and non-destructive tests for the determination of the in-plane properties of chopped strand mat reinforced epoxy laminates, Proceedings of trhe $3^{\text {rd }}$ International Conference on NDT, NDT in Antiquity and nowadays skills-applications-innovations, Hellenic Society for NDT, Chania-Crete, Greece, 2003, 208-216.

[6] Prassianakis I.N., Kytopoulos V.N., Sideridis E. Investigation of mechanical properties of epoxy resins and random fibre epoxy systems by means of destructive and non-destructive tests, accepted for publication in Int. Journ. of Materials and Product Technology, special issue NDT \& FPT.

[7] Wang J.Z., Dillard D.A., Wolcott M.P., Kamke F.A., Wilkes G.L.: Transient moisture effects in fibres and composite materials, Journal of Composite Materials, Vol. 24, No. , 1990, 994-1009.

[8] Tenneg D.R., Unnam J.: Analytical prediction of moisture absorption in composites, Journal of Aircraft, Vol. 15, No. 3, 1978, 148-154.

[9] Shen Chi-Hung, Springer G.S.: Moisture absorption and desorption of composite materials, Journal of Composite Materials, Vol. 10, January, 1976, 2-20.

[10] Springer G.S.: Moisture content of composites under transient conditions, Journal of Composite Materials, Vol. 11, January, 1977.

[11] Cai L.W., Weitsman Y.: Non-Fickian moisture diffusion in polymeric Composites, Journal of Composite Materials, Vol. 28, No 2, 1994, 130-154.

[12] Vanlandingham M.R., Eduljee R.F., Gillespie J.W. Jr.: Moisture diffusion in epoxy systems, Journal of Applied Polymer Science, Vol. 71, 1999, 787-798.

[13] Zhou J., Lucas J.P.: The effects of water environment on anomalous absorption behaviour in graphite/epoxy composites, Composite Science and Technology, Vol. 53, 1995, 57-64. 